\title{
Necesidades identificadas por egresados de medicina durante su transición preclínica-clínica
}

\section{Needs identified by medical graduates during their preclinical-clinical transition}

Recibido 25/09/2020

Amy Lucila Castro-de-Reyes

Universidad de San Carlos de Guatemala investigarprimeroversion2@gmail.com

https://orcid.org/0000-0003-2887-1345

Maribel Valenzuela

Universidad de San Carlos de Guatemala mari_val70@yahoo.com

https://orcid.org/0000-0002-6124-9112

Aceptado 18/01/2021

\section{Referencia}

Castro-de-Reyes, A. L. y Valenzuela, M. (2021). Necesidades identificadas por egresados de medicina durante su transición preclínica-clínica. Revista Docencia Universitaria, 2(1), 10-22. https://doi.org/10.46954/revistadusac.v2i1.20

\section{Resumen}

El paso de la vida académica intra-aula a la práctica hospitalaria es conocido como transición preclínica-clínica (TPC), es reconocido como traumático y potencialmente lesivo a la salud mental de los estudiantes de medicina, es parte de la formación de la Facultad de Ciencias Médicas de la USAC de Guatemala (FacMed). Exponer las necesidades identificadas por los egresados en 2020, cuando pasaron por la TPC sirve como marco de referencia para la mejora continua de los procesos educativos, estableciendo las necesidades psicofisiológicas y educativas que identificaron. La investigación fue cuantitativa, descriptiva, transversal, muestreo no probabilístico por bola de nieve. 54 egresados brindaron su consentimiento informado para participar en el estudio, se utilizó el cuestionario electrónico NIEM-TPC2020. 55\% 
de las respuestas fueron de mujeres, 75\% vivió la primera rotación en 2017, el 27.78\% lo realizó en el Hospital General San Juan de Dios, la primera rotación fue Medicina Interna (59.26\%). Necesidades psicofisiológicas más identificadas: proteger su salud mental, sus objetos personales, el reconocimiento y valoración a su dignidad, satisfacer necesidades básicas. Necesidades educativas identificadas: inducción al hospital, acompañamiento del docente, preparación administrativa, afrontamiento de la muerte de sus pacientes, estrategias motivadoras del aprendizaje.

\section{Abstract}

The transition from intra-classroom academic life to hospital practice is known as preclinical-clinical transition (PCT), it is recognized as traumatic and potentially harmful to the mental health of medical students, it is part of the training of the Faculty of Medical Sciences of the USAC of Guatemala (FacMed). Exposing the needs identified by the graduates in 2020, when they went through the TPC serves as a frame of reference for the continuous improvement of educational processes, establishing the psychophysiological and educational needs that they identified. Quantitative, descriptive, cross-sectional research, non-probabilistic snowball sampling. 54 graduates gave their informed consent to participate in the study, the NIEM-TPC2020 electronic questionnaire was used. 55\% of the responses were from women, $75 \%$ experienced the first rotation in 2017, 27.78\% did it at General San Juan de Dios Hospital, the first rotation was Internal Medicine (59.26\%). Most identified psychophysiological needs: protect their mental health, their personal belongings, the recognition and appreciation of their dignity, satisfy basic needs. Identified educational needs: hospital induction, teacher accompaniment, administrative preparation, coping with the death of their patients, motivational learning strategies.

\section{Introducción}

En el proceso de formación de los futuros médicos, la práctica hospitalaria es fundamental para el desarrollo de las competencias que les permitan desenvolverse como profesionales de la salud. Esto genera el acercamiento del estudiante a los procesos de la vida, en el aspecto biopsicosocial, al dolor, al sufrimiento humano, y al proceso de la muerte, impacta en las creencias, valores, idea propia del mundo, interpretación del pasado y expectativas de futuro (Cabrera López, 2005, p. 1).
Palabras clave: egresados de medicina, necesidades educativas, necesidades fisiológicas, necesidades psicológicas, transición preclínica-clínica.

Keywords: medical graduates, educational needs, physiological needs, psychological needs, preclinical-clinical transition. 
Durante la práctica hospitalaria, el estudiante de medicina desarrolla la capacidad de recuperación ante estos procesos, para transformar en oportunidades de aprendizaje las experiencias vividas. En la FacMed, los estudiantes afrontan en el cuarto año de la carrera, el paso de las aulas universitarias a la práctica hospitalaria, evento conocido como Transición pre Clínica-Clínica (TPC).

Según Cabrera (2005) "los cambios y transiciones son componentes inherentes a la vida humana" diferenciados en que un cambio es una "modificación/sustitución de un hecho o elemento por otro" con duración corta y drástica, con procesos externos de forma puntual, mientras que la transición implica procesos con duración más prolongada, cambios, es gradual y siempre conlleva procesos psicológicos (internos) (p. 83).

El estudio planteado se realizó porque los diversos eventos en la vida y el ambiente del estudiante de medicina que experimenta la TPC, pueden resultar traumáticos y lesionar su salud mental, y generar consecuencias de dolor, inseguridad, depresión, ansiedad y menoscabo de su salud mental (Díaz, 2010, p. 6).

Pese a que se hacen esfuerzos por dar a los estudiantes orientación y capacitación referente a la parte clínica propiamente dicha, aún hay vacíos de preparación que les permitan adaptarse no solo en el aspecto académico sino también al entorno laboral con ajustes psicosociales para transitar adecuada y progresivamente por este evento de sus vidas.

Estableciendo que una necesidad es la distancia que hay entre la situación como una persona la está viviendo y como le gustaría vivirla (Vázquez y Valbuena, 2009, p. 2) y que estas varían según el contexto (Cavalcanti, et al., 2020, p. 2) era importante detectarlas a fin de establecer información que permita convertirse en marco de referencia para la mejora continua de los procesos de formación, y evitar que el proceso sea adverso para los estudiantes.

El aprendizaje clínico, es considerado parte de los aprendizajes en contextos de trabajo, sustentado en teorías socio-culturales, como el aprendizaje situado y las comunidades de práctica (Bitrán, et al., 2014, p. 724); trabajo es toda actividad que conlleva retribución, pero, en calidad de aprendiz, se reconoce que es trabajo no remunerado, es decir aquel por el que no se obtiene salario (Ariadna, 2020, p. 1). 
Se considera que este tipo de trabajo carece de regulación legal, horario regulado, el cual suele ser incluso mayor al tiempo a una jornada laboral normal, entre otras características que están en concordancia con las actividades de los estudiantes cuando desarrollan su práctica, es de esperarse que existan necesidades no satisfechas durante este período. Ibídem.

Al llegar a su TPC, el estudiante, se convierte en trabajador de un momento a otro, y está más preocupado "por cumplir las tareas que le dejan en el hospital, por cumplir los turnos", que, por aprender, suele ver a los estudiantes, "realizar el trabajo que corresponde a paramédicos y enfermeros. Ventilar a los pacientes, trasladar muestras de laboratorio, llevar a los enfermos en silla de ruedas o en camilla a Rayos X" situación que se ha dado en los últimos años en los hospitales escuela, sin oportunidad de intervención o mejora (Ola, 2020, párr. 8, 9).

Para resguardar a los estudiantes de la pandemia de COVID-19 que afectó en el año 2020, la FacMed suspendió las actividades prácticas, no se pudo contactar a estudiantes que estuvieran atravesando su TPC durante ese año, por lo que se tomó en cuenta las experiencias que los egresados de la carrera de medicina en el año 2020 pudieran recordar.

El objetivo de éste artículo es exponer las principales necesidades psicofisiológicas y educativas identificadas, en la TPC, por 54 egresados en 2020, de la carrera de medicina del CUM-USAC Guatemala.

\section{Materiales y métodos}

Estudio cuantitativo, descriptivo, transversal. Se diseñó el cuestionario NIEM-TPC2020. Se realizó muestreo no probabilístico, por bola de nieve, obteniéndose el consentimiento de 54 egresados de la carrera de Medicina, que realizaron su primera práctica hospitalaria en los hospitales escuela que tienen convenio con la FacMed, en la unidad didáctica: externado de Medicina o de Cirugía, el instrumento fue validado a través de consulta a expertos, evaluando constructo, contenido, redacción y estilo, avalado por el Instituto de Investigaciones Humanísticas. Se realizó prueba piloto con participantes que aceptaron colaborar; cuyas sugerencias modificaron y mejoraron el instrumento. Las necesidades fueron evaluadas según los objetivos establecidos, aplicando escalas valorativas tipo Likert, a) los resultados de cada pregunta fueron recategorizados a los punteos que se le asignaron a cada opción, b) se sumaron las cantidades y se promediaron obteniéndose un valor en los rangos de 1 a 5 , los promedios menores a 3 se consideraron como las necesidades identificadas. 


\section{Resultados}

Tabla No. 1 Datos generales y académicos de los participantes

Edad y sexo de los participantes

\begin{tabular}{|c|c|c|c|c|c|c|}
\hline Edad & $\mathbf{N}$ & $\%$ & $\mathbf{F}$ & $\%$ & $\mathbf{M}$ & $\%$ \\
\hline $21-23$ & 3 & $(5.56)$ & 1 & (3.33) & 2 & $(8.00)$ \\
\hline $24-26$ & 37 & $(68.52)$ & 20 & $(66.67)$ & 17 & $(68.00)$ \\
\hline 27-29 & 12 & $(22.22)$ & 7 & $(23.33)$ & 5 & $(20.00)$ \\
\hline 30 o mayor de 30 & 2 & (3.70) & 2 & $(6.67)$ & 0 & $(0.00)$ \\
\hline Total & 54 & $(100.00)$ & 30 & $(100.00)$ & 24 & $(100.00)$ \\
\hline \multicolumn{7}{|c|}{ Año de primera práctica hospitalaria } \\
\hline Año & $\mathbf{N}$ & $\%$ & $\mathbf{F}$ & $\%$ & $\mathbf{M}$ & $\%$ \\
\hline antes 2015 & 1 & $(1.85)$ & 1 & (3.33) & 0 & $(0.00)$ \\
\hline 2015 & 4 & (7.4) & 2 & (6.67) & 2 & $(8.00)$ \\
\hline 2016 & 7 & (12.96) & 1 & (3.33) & 6 & $(24.00)$ \\
\hline 2017 & 41 & (75.93) & 25 & (83.33) & 16 & $(64.00)$ \\
\hline 2018 & 1 & (1.85) & 1 & (3.33) & 0 & $(0.00)$ \\
\hline Total & 54 & (100.00) & 30 & $(100.00)$ & 24 & (100.00) \\
\hline \multicolumn{7}{|c|}{ Hospital en que realizó la primera práctica hospitalaria } \\
\hline Hospital & $\mathbf{N}$ & $\%$ & $\mathbf{F}$ & $\%$ & $\mathbf{M}$ & $\%$ \\
\hline General San Juan de Dios & 15 & $(27.78)$ & 9 & $(30.00)$ & 6 & $(25.00)$ \\
\hline Roosevelt & 14 & $(25.93)$ & 6 & $(20.00)$ & 8 & (33.33) \\
\hline IGSS & 12 & $(22.22)$ & 6 & $(20.00)$ & 6 & $(25.00)$ \\
\hline Regional de Escuintla & 5 & (9.26) & 4 & $(13.33)$ & 1 & $(4.17)$ \\
\hline Regional de Cuilapa & 4 & (7.41) & 2 & (6.67) & 2 & $(8.33)$ \\
\hline Nacional de Antigua & 2 & $(3.70)$ & 2 & (6.67) & 0 & $(0.00)$ \\
\hline Otro: & 2 & (3.70) & 1 & (3.33) & 1 & $(4.17)$ \\
\hline & 54 & $(100.00)$ & 30 & $(100.00)$ & 24 & $(100.00)$ \\
\hline \multicolumn{7}{|c|}{ Primera rotación realizada } \\
\hline Rotación & $\mathbf{N}$ & $\%$ & $\mathbf{F}$ & $\%$ & $\mathbf{M}$ & $\%$ \\
\hline Medicina Interna & 32 & $(59.26)$ & 18 & $(60.00)$ & 14 & $(58.33)$ \\
\hline Cirugía & 22 & $(40.74)$ & 12 & $(40.00)$ & 10 & $(41.67)$ \\
\hline Total & 54 & $(100.00)$ & 30 & $(100.00)$ & 24 & $(100.00)$ \\
\hline
\end{tabular}

Abreviaturas: F= Femenino, M= Masculino; IGSS: Instituto Guatemalteco de Seguridad Social zona 9 y 7-19.

Fuente: propia, con datos obtenidos en el cuestionario en línea NIEM-TPC2020, respondido por 54 graduados en 2020. 
Figura No. 1 Necesidades psicofisiológicas identificadas por egresados en 2020, durante su transición preclínica-clínica

\section{Necesidades psicofisiológicas identificadas por egresados en 2020 durante su transición preclínica-clínica}

Recursos humanos de la facultad, para cuidar de mi

Lugar donde pudiera resguardar mis objetos

Realización de actividades de estudiante de medicina

Lugar destinado a los estudiantes para descansar

Lugar donde conseguir uniformes para sala de

Valoración como persona, con buena reputación, por

Reconocimiento hacia mi persona por la labor

Seguridad de mi integridad física dentro de las

Realizar actividades con la asesoría o supervisión

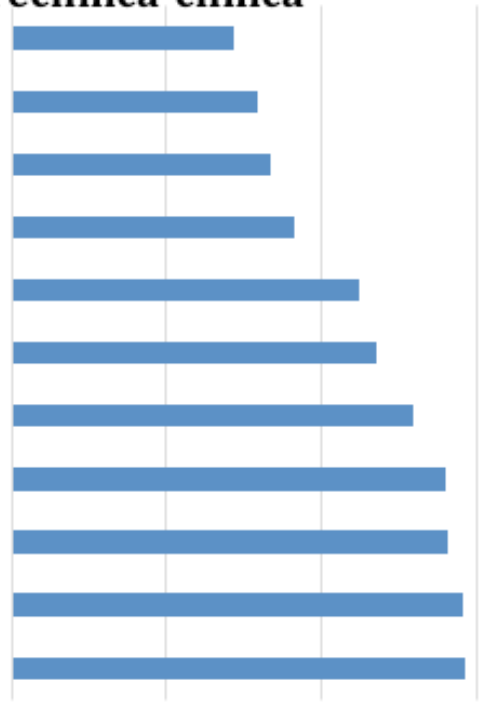

0

1

2

3

Fuente: propia, con datos obtenidos en el cuestionario en línea NIEM-TPC2020

La figura 1 evidencia que los recursos humanos para cuidar de la salud mental fue la principal necesidad psicológica identificada, la dignidad como estudiante de medicina al realizar actividades destinadas a otros trabajadores de salud fue la segunda, la valoración como persona aunada a la buena reputación por el personal paramédico la tercera y el reconocimiento a la labor realizada la cuarta necesidad psicológica identificada.

En esta misma figura se establece que en el aspecto fisiológico, la principal necesidad detectada fue la seguridad para resguardar sus pertenencias, en segundo lugar, encontrar un sitio para descansar, seguido de los recursos para cuidar de su salud como uniformes y atención médica en caso necesario. 
Figura No. 2 Necesidades psicofisiológicas identificas por egresados durante TPC por sexo

\section{Necesidades psicofisiologicas identificadas durante la transición preclínica-clínica, por sexo}

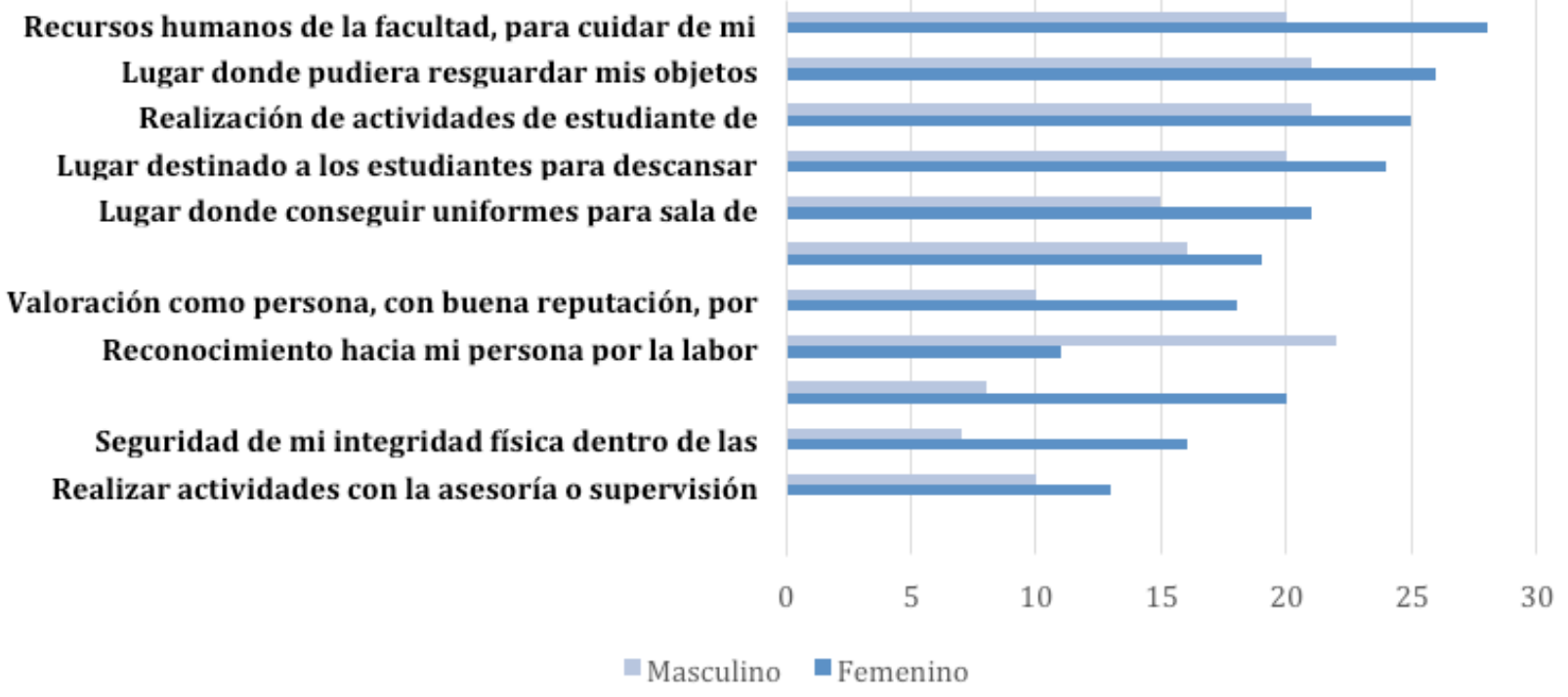

Fuente: propia, con datos obtenidos en el cuestionario en línea NIEM-TPC2020

En la figura No. 2 se establece que las necesidades fueron principalmente identificadas por el sexo femenino, a excepción de la necesidad de reconocimiento a la labor realizada, que fue más identificada por el sexo masculino. 
Figura No. 3 Necesidades educativas identificadas por egresados en 2020 durante su transición preclínica-clínica

\title{
Necesidades educativas identificadas por egresados en 2020 durante su transición preclínica-clínica
}

\begin{abstract}
Visita guiada por la facultad a las instalaciones del Acompañamiento por parte de mis profesores de la Enseñanza de habilidades para realizar las labores Preparación para enfrentar el evento de la muerte Médico responsable de los estudiantes, explicaba Enseñanza de habilidades clínicas y técnicas para Preparación y acompañamiento, guiado, asesorado y Acompañamiento de los otros actores del ámbito Estrategias de enseñanza que motivaban el Integración del conocimiento teórico y analítico de Apoyo de las autoridades del hospital escuela, al Aclaración de derechos y obligaciones de los
\end{abstract}
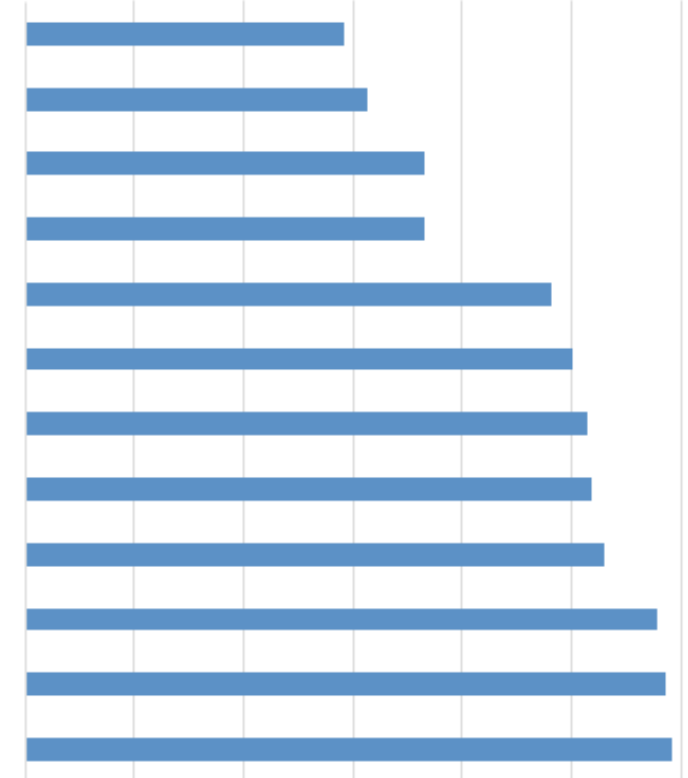

Fuente: datos obtenidos en el cuestionario en línea NIEM-TPC2020

En la figura 3 es evidente que la principal necesidad educativa identificada fue la inducción laboral por medio de una visita guiada a las instalaciones del hospital escuela por parte de los responsables de la FacMed, seguida del acompañamiento por parte de los profesores para afrontar la muerte de los pacientes, y en tercer lugar la enseñanza de las habilidades para realizar labores administrativas. 


\section{Discusión}

Se establecieron once necesidades psicofisiológicas para los egresados, de las cuales cuatro de ellas fueron psicológicas y siete fisiológicas.

En acuerdo con Díaz (2010) y Rodríguez, et al. (2014), quienes afirman que los estudiantes de medicina encuentran la carrera muy estresante desde el inicio y su salud mental está en constante riesgo en el aspecto psicológico, los datos reflejan que la principal necesidad es el cuidado de su salud mental, coincide con la afirmación de Gouveia et al. (2005) de que "cuanto mayor sea el grado de satisfacción de las necesidades, mejor será la salud mental del individuo", (p. 3), por tanto, al no satisfacer las necesidades de la base de la pirámide, no es posible cuidar de las relativas al nivel superior.

La segunda necesidad en el aspecto psicológico se encuentra la dignidad al realizar actividades destinadas al estudiante de medicina y no al reemplazo a otros trabajadores, como lo afirmaba el artículo de Ola (2020), en relación a lo anterior, para Chávez-Rivera, et al. (2016) "la asignación de una carga de trabajo académica o clínica de manera excesiva o inapropiada", es considerado maltrato académico, habría que revisar en futuras investigaciones si estas actividades asignadas a los estudiantes perpetúan un tipo de maltrato soslayado (p. 806).

A continuación, se identifica la infravaloración como persona, mala reputación por parte de personal paramédico, y la más identificada por el sexo masculino, ausencia de reconocimiento a la labor realizada. Se sabe que los estudiantes que experimentan rechazo e infravaloración o reconocimiento a su labor realizada perciben "respuestas que utilizan los mismos circuitos neuronales involucrados en el dolor físico" (Cavalcanti, y otros, 2020, p. 3), no es extraño que las lesiones a su salud mental se manifiesten no solo por no satisfacer las necesidades fisiológicas, sino por sentir directamente un ataque a su psiquis.

Entre las necesidades fisiológicas, los datos reflejan que garantizar seguridad de sus pertenencias, lugares adecuados para descansar y tener horas de sueño suficientes para recuperarse, lugar donde recibir atención médica, donde conseguir y consumir alimentos, seguridad de su salud física, seguridad de su integridad física porque había seguridad en el hospital-escuela, y de realizar procedimientos bajo asesoría y 
supervisión que no implicara riesgos para su salud fueron identificadas. Si bien es cierto en la pirámide de Maslow, las necesidades fisiológicas básicas se encuentran ocupando mayor espacio y se imponen a las demás (Vázquez Muñoz y Valbuena de la Fuente, 2009), no se espera que el hospital-escuela no les garantice la satisfacción de estas necesidades de los niveles inferiores.

En el aspecto académico, doce necesidades son identificadas por los egresados. En primera instancia está la carencia de una inducción laboral, a través de una visita guiada a las instalaciones del hospital escuela, por parte de la FacMed, contrario a lo encontrado en el estudio de Rashid-Doubell y colaboradores (2019) que mencionan haber recibido en la primera práctica clínica una inducción adecuada y oportuna que incluyó detalles de su papel y responsabilidades.

En segundo lugar, se encuentra la necesidad de acompañamiento de los profesores para enfrentar el evento de la muerte de sus pacientes, 45 de los 54 encuestados refirieron no recibir dicho acompañamiento, situación para la que no se sentían preparados concordando con el estudio de Henriquez et al. (2014) la falta de estrategias de acompañamiento por parte de docentes encargados coincide con lo afirmado por Acuña y López (2014), como evidencia del abandono al estudiante en estos momentos de aprendizaje desde antes de la TPC, durante y después de ella, situación que potencia la lesión a su salud mental.

En la parte académica no son los estudios previos que les permitan la "comprensión e interpretación de exámenes de laboratorio, procedimientos manuales, instrumentales, formulación de plan diagnóstico, terapéutico y educacional" de los que carecen los estudiantes como afirma (Cayax, 2006, p. 11), sino de la preparación para el llenado de papelería administrativa, médico responsable que explique los eventos de salud, que les enseñe habilidades clínicas y técnicas para afrontar la práctica con acompañamiento y asesoramiento profesional, y de parte de otros actores como los residentes, estrategias motivadoras, y apoyo de las autoridades así como la aclaración de sus derechos y obligaciones, lo que coincide con el estudio "lanzado al fondo" de (Rashid-Doubell et al., 2019), que deja a los practicantes con incertidumbre sobre su papel y responsabilidades que deben realizar. 
Es recomendable que la FacMed tome en cuenta que para lograr un aprendizaje significativo durante la práctica clínica en el contexto hospitalario, debe realizarse con acompañamiento integral, apoyo de otros, protección a los estudiantes, garantía de que sus necesidades psicofisiológicas estén cubiertas, generando automotivación, con estrategias de enseñanza aprendizaje, fomento del aprendizaje reflexivo, cuidado de la salud mental, abordaje y afrontamiento de la muerte de los pacientes y su repercusión en el desempeño profesional, considerando ciclos básicos preclínicos y clínicos en forma integrada y no segmentada, visitas continuas al hospital, para conocerlo, familiarizarse con la papelería administrativa, asistir a sesiones clínicas, permitiendo al futuro practicante sentirse más habituado con el entorno en el que ha de trabajar.

\section{Referencias}

Acuña, L., Y López, E. (2014). Sufrimiento emocional del estudiante de medicina en el inicio de la práctica hospitalaria ante la muerte de un paciente. Guatemala, Guatemala, Guatemala: Facultad de Ciencias Médicas. Obtenido de http://psicologia.usac.edu. gt/?page_id=137317

Ariadna, I. (2020). Economipedia.com. Obtenido de Trabajo no remunerado: Obtenido de https://economipedia.com/ definiciones/trabajo-no-remunerado.html

Bitrán, M., Zúñiga, D., Leiva, I., Calderón, M., Tomicic, A., Padilla, O., Y Riquelme, A. (2014). ¿Cómo aprenden los estudiantes de medicina en la transición hacia el ciclo clínico? Estudio cualitativo de las percepciones de estudiantes y docentes acerca del aprendizaje inicial de la clínica. Rev Med Chile, 723731. Obtenido de https://scielo.conicyt.cl/pdf/rmc/v142n6/ art06.pdf

Cabrera, G. (2005). La transición PreClínico-Clínico en la carrera de Medicina. (T. D. Educación, Ed.) Barcelona, España: Universidad de Barcelona. Obtenido dehttps://drive.google. com/file/d/1lo5cU7gtdUvnXeBjhkO2OWaDGVbMAPh/ view?usp=sharing

Cavalcanti, T. M., Gouveia, V. V., de Medeiros, E. D., Tailson, E. M., Hysla, M. M., Y Moizeís, H. B. (2020). Hierarquia das Necessidades de Maslow: Validacao de um Instrumento. 
Psicología: Ciencia e Profissao. Obtenido de http://

www.scielo.br/scielo.php?script=sci_arttextYpid=S1414-

98932019000100162Ylang=es\#B7

Cayax, L. I. (2006). El proceso formativo de los tres primeros años de la carrera de medicina y sus efectos en el desempeño de los estudiantes en la práctica hospitalaria. (C. U. Occidente, Ed.) Quetzaltenango, Quetzaltenango, Guatemala: Universidad de San Carlos de Guatemala. Obtenido de https://drive. google.com/file/d/1G4Pbxr8QLGACDAiBE-b2fTqfGXXtutRz/ view?usp=sharing

Chávez-Rivera, A., Ramos-Lira, L., Y Abreu-Hernández, L. F. (2016). Una revisión sistemática del maltrato en el estudiante de medicina. GacMedMex, 152, 796-811. Obtenido de https:// www.anmm.org.mx/GMM/2016/n6/GMM_152_2016_6_796811.pdf

Díaz, Y. (2010). Estrés académico y afrontamiento en estudiantes de Medicina. Rev Hum Med. Obtenido de http://scielo.sld.cu/ pdf/hmc/v10n1/hmc070110.pdf

Gouveia, V., Alves, G., Y de Oliveira, E. B. (2005). Medindo a satisfação com a vida dos médicos no Brasil. Jornal Brasileiro de Psiquiatria, 298-305. Obtenido de https://www.researchgate. net/publication/279194945_Medindo_a_satisfacao_com_a_ vida_dos_medicos_no_Brasil

Ola, A. L. (12 de febrero de 2020). Preparan cambio de horario para estudiantes de Medicina en hospitales escuela. Prensa Libre. Obtenido de https://www.prensalibre.com/tema/facultad-demedicina/

Rashid-Doubell, F., Y Doubell, T. (2019). Lanzado al fondo: la experiencia de los graduados de la educación médica transnacional occidental en transición hacia la práctica clínica del Medio Oriente. International Journal of Higher Education, 36,38-47. Obtenido de http://www.sciedu.ca/journal/index. php/ijhe/article/view/16288

Rodríguez, M., Sanmiguel, M., Muñoz, A., Y Rodríguez, C. (2014). El estrés en estudiantes de medicina al inicio y final de su formación académica. Revista Iberaméricana de Educación (66), 105-122. Obtenido de https://rieoei.org/historico/ documentos/rie66a07.pdf 
Vázquez Muñoz, M., Y Valbuena de la Fuente, F. (2009). infonegociacion.net. Recuperado el 18 de 04 de 2020, de La Pirámide de Necesidades de Abraham Maslow. Obtenido de http://www.infonegociacion.net/pdf/piramide-necesidadesmaslow.pdf

\section{Agradecimientos}

A la Universidad de San Carlos de Guatemala, a la Facultad de Humanidades, al Maestro Bidel Méndez, a la Dra. Maribel Valenzuela, asesora, a los egresados de Medicina del año 2020, a los Doctores Magda Velásquez (CICS), Dorian Ramírez (CICS), José Antonio Álvarez, Nehemías Calel, María Fernanda Monterroso, Angélica Rodríguez.

\section{Sobre las autoras}

\section{Amy Lucila Castro-de-Reyes}

Es Médico y cirujano de la FacMed USAC Guatemala, auditora en salud por la UNINCCA de Colombia, profesora titular d e Fundamentos d e la investigación Científica de la Fac Med. Miembro de las aso ciaciones de Médicos Escritores de Guatemala y Latinoamérica. Investigadora en salud, galardonada en certámenes nacionales e internacionales de escritura científica y literaria. Estudiante de la Maestría en Do cencia Universitaria de la FaHUSAC Guatemala.

\section{Maribel Alejandrina Valenzuela Guzmán}

Es Doctora en Educación, trabaja en la Universidad de San Carlos, como docente a nivel de Maestría y Doctorado.

\section{Copyright (c) Amy Lucila Castro-de-Reyes y Maribel Alejandrina Valenzuela Guzmán}

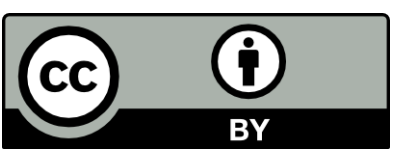

Este texto está protegido por una licencia Creative Commons 4.0.

Usted es libre para compartir, copiar y redistribuir el material en cualquier medio o formato y adaptar el documento, remezclar, transformar y crear a partir del material para cualquier propósito, incluso comercialmente, siempre que cumpla la condición de atribución: usted debe reconocer el crédito de una obra de manera adecuada, proporcionar un enlace a la licencia, e indicar si se han realizado cambios. Puede hacerlo en cualquier forma razonable, pero no de forma tal que sugiera que tiene el apoyo del licenciante o lo recibe por el uso que hace. 